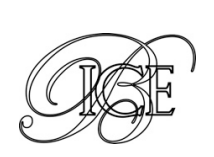

Rubén Ruiz Ramas*

\title{
LA DIVERSIFICACIÓN DEL PODER EN LOS ESTADOS NEOPATRIMONIALISTAS DE ASIA CENTRAL
}

Tras más de dos décadas de experiencia postsoviética en Asia central, el continuum entre autoritarismo y democracia liberal no captura la heterogeneidad de trayectorias políticas existentes en la región. En consecuencia, los últimos años dejan una renovación teórica orientada, primero, a conocer con mayor profundidad los diferentes subtipos de autoritarismos y, segundo, a otorgar relevancia a las instituciones informales en Estados categorizados como neopatrimoniales. En este artículo se trabaja desde la perspectiva de la Construcción del Estado (Statebuilding) para distinguir en los Estados neopatrimoniales tres niveles de diversificación del poder basados en dos rasgos principales: la estructura estatal de toma de decisiones en el Gobierno central y el grado de fragmentación territorial del poder.

Palabras clave: Asia central, neopatrimonialismo, diversificación del poder, construcción del Estado, fragmentación territorial.

Clasificación JEL: O17, F51, F54, Z13.

\section{Introducción}

Tras más de dos décadas de experiencia postsoviética el paradigma de la transición a la democracia liberal ha sido superado. Es evidente que la democracia no es una meta perseguida por la mayor parte de las élites políticas y, por tanto, el continuum entre autoritarismo y democracia liberal no captura la heterogeneidad de trayectorias políticas existentes en la región (Carothers, 2002; Jones Luong, 2004; Cummings, 2012; Susan Stewart et. al., 2012). En consecuencia, los últimos años dejan una renovación teórica orientada, primero, a conocer con mayor profundidad los diferentes subtipos de autoritarismos (Møller y Skaaning, 2010), y segundo, a otorgar relevancia a las instituciones informales en Estados categorizados como neopatrimoniales (Wilson, 2005; Hale, 2006 y 2011;

\footnotetext{
* Departamento de Ciencia Política y de la Administración, UNED. Versión de abril de 2015.
}

Iljamov, 2007; Collins, 2009; Fisun, 2007; Isaacs, 2010; Guliyev, 2011; Kononenko y Moshes, 2011; Ruiz Ramas, 2013).

Los Estados neopatrimonialistas tienen unas características comunes pero también se diferencian en otras, es decir, existe heterogeneidad de trayectorias neopatrimoniales. La mayoría de intentos por establecer una tipología permanente combinan la estructura de autoridad del Estado (desde la racionalidad burocrática a la dominación patrimonial) con el nivel de competición política (liberalización y participación) en un régimen político dado, es decir, la suma de las perspectivas de Weber y Dahl (Erdmann y Engel, 2007; Von Soest, 2010; Fisun, 2007; Guliyev, 2012). Sin embargo, entre los Estados centroasiáticos, mientras solo Kirguistán ha experimentado variaciones en el eje autoritarismo-democracia liberal, se ha vivido y se vive una intensa competición por ejercer la autoridad estatal (state authoritymaking), por posicionarse en el reparto del poder a nivel central (power-sharing) y por disputar la $\triangleright$ 
autoridad efectiva en las regiones a las instituciones centrales. Siendo la construcción del Estado (State-building) un proceso incompleto en Asia central, cada país tiene sus propias dinámicas y contiendas en la competición por la autoridad estatal y en todas aquellas arenas donde reside de facto el poder.

Por lo tanto, otorgando relevancia a las instituciones informales este artículo propone un cambio de énfasis, desde los paradigmas clásicos de los regímenes políticos y las transiciones, hacia el marco menos explorado en la Ciencia Política de la construcción del Estado (Grzymala-Busse y Jones Luong, 2002; Ganev, 2005; Cummings, 2012). Para analizar la heterogeneidad en los Estados neopatrimoniales se distinguirán tres niveles de diversificación del poder basados en la agregación de dos rasgos principales: la estructura estatal de toma de decisiones en el Gobierno central y el grado de fragmentación territorial del poder. Esto es, las dos fases del proceso de formación de la autoridad estatal: la estructura de toma de decisiones, principalmente en el Gobierno central en Estados unitarios; y el proceso de implementación de las normas resultantes en los estratos local y regional del Estado. En este sentido, para explicar la diversificación del poder en Asia central hay que concentrar parte del análisis en aquellos impasses en la aplicación de la autoridad central que provocan una «migración de la autoridad» informal a los agentes e instituciones locales.

\section{Neopatrimonialismo y heterogeneidad de trayectorias}

De manera sintética, por Estado neopatrimonial se entiende un Estado donde coexisten e interactúan lógicas de acción formales (burocráticas) e informales (patrimoniales). El concepto tiene su origen en la distinción de Weber de distintos tipos de autoridad (tradicional, carismática, y burocrática o legal-racional), habiendo sido desarrollado en su forma moderna por investigadores interesados en los Estados poscoloniales africanos
(Eisenstadt, 1978; Snyder, 1992; Médard, 1982; Bratton y van de Walle, 1997; Erdmann y Engel, 2006; Erdmann, 2012). Cuando se habla de neopatrimonialismo no identificamos un tipo particular de régimen político, sino un conjunto de prácticas institucionalizadas en los agentes e instituciones estatales que no pueden ser fácilmente eliminadas o desafiadas por nuevos actores ya que están históricamente enraizadas (Von Soest, 2010).

Estas instituciones pueden relacionarse con aspectos políticos (Gobiernos fuertes y con una toma de decisiones concentrada, clientelismo, patronazgo, incertidumbre institucional, reclutamiento nepotista, lealtad a los superiores) o económicos (corrupción, apropiación ilícita de bienes privados o activos del Estado, rentismo), desarrollados bien en las estructuras de Gobierno bien en otros puntos de la Administración del Estado.

Un régimen personalista equipado con una concentración informal de poder, la apropiación privada de la arena pública, un clientelismo sistémico y el uso de los recursos del Estado para la legitimación política de particulares, son las instituciones informales primigenias relacionadas con un Estado neopatrimonial (Bratton y van de Walle, 1997). No obstante, se deben destacar otros rasgos institucionales claves. En primer lugar, la incertidumbre institucional. La persistencia del neopatrimonialismo en un Estado dado depende de una constante actualización de las interacciones entre las instituciones formales e informales, la cual únicamente se puede alcanzar por medio de la incertidumbre institucional. Ésta permite moldear instituciones políticas formales, como puede ser el conjunto de normas que operan en unas elecciones o en una institución reguladora en una economía de mercado, para servir a los intereses de las autoridades o de personas concretas (Ruiz Ramas, 2013). La incertidumbre también refuerza las relaciones clientelares con la élite dirigente al interpretarse que son la forma más segura de proveer predictibilidad a los distintos actores sociales (Timm, 2010).

En segundo lugar, los Estados neopatrimoniales son vulnerables ante la fragmentación del poder $D$ 
político. A pesar de que el personalismo es una característica central de este tipo de dominación, rara vez los escenarios neopatrimoniales funcionan como consistentes sistemas verticales. Para funcionar, los Estados necesitan incorporar mecanismos de abajo a arriba, y los gobernantes contar con la lealtad y colaboración de intermediadores o brokers locales. Conflictos, exigencias, negociaciones y compromisos en la sombra son el día a día en las relaciones entre los actores del centro y de la periferia. La implementación de las políticas aprobadas por el centro en las regiones queda frecuentemente al margen de la capacidad de control del centro. En muchas ocasiones, de hecho, «el desafío central para la autoridad neopatrimonial es prevenir una descentralización incontrolada de las estructuras de poder» (Timm, 2010).

En tercer lugar, la política en estos Estados es una arena flexible donde los elementos programáticos de fractura (cleavages) son fácilmente reemplazables y los enemigos hoy irreconciliables pueden compartir mañana coaliciones en el poder (power-sharing coalitions). Esta situación es favorecida por el rol central de las redes informales, las cuales como tipo de vehículo de acción colectiva son tan importantes o más que los propios partidos políticos. Existe un amplio consenso en la academia acerca de la centralidad de las redes informales en la política postsoviética, pero no lo hay respecto a su naturaleza. En los estudios sobre Asia central, destacan cuatro conceptuaciones: el clan-tribal como identidad subnacional (Schatz, 2005; Collins, 2006); la red informal como asociación regional de élites (Jones Luong, 2002; Iljamov, 2007); la red informal como entidad personalista y local, no regional, en países como Kirguistán (Radnitz, 2005, 2006, 2010; Sjöberg, 2011; Ruiz Ramas, 2013), y finalmente para casos como Rusia, Ucrania o Kazajstán los finansovo-promyshlennye gruppy o Bussiness-Industrial Groups (FPG), redes de élites económicas de alcance global controlando corporaciones financieras, industriales y energéticas además de significativas parcelas del poder político (Cummings, 2005; Balmaceda, 2008; Ostrowski, 2009; Junisbai, 2010; Kononenko y Moshes, 2011).
Ciertamente, las características de las redes predominantes varían de un país a otro al divergir las condiciones económicas, políticas y sociales en las que se han desarrollado. Con todo, los estudios más recientes rechazan que los clanes-tribales tradicionales mantengan su influencia en la esfera política. Por el contrario, se sostiene la creciente consolidación de camarillas clientelistas alimentadas más por el dinero y el intercambio de favores que por ancestrales vínculos sanguíneos, estructuradas mediante conexiones personales vitales y profesionales en vez de por el parentesco e identidades subnacionales (lljamov, 2007; TunçerKilavuz, 2011; Radnitz, 2010; Cummings, 2012; Laurelle, 2012; Ruiz Ramas, 2013). Las redes informales hoy tienden a ser flexibles en composición, temporales y no grupos corporativos. Organizadas en una estricta jerarquía, compiten entre sí por recursos limitados pero son capaces de coordinar acciones colectivas en coaliciones transitorias. De hecho, en un mismo país pueden coexistir y colaborar en redes de distinta naturaleza, tales como camarillas de élites operando solo en los estratos más altos del poder político junto a redes de ámbito local o regional capaces de movilizar distritos y actuar así como agentes influyentes (power brokers) en favor de una opción influyente a nivel central.

No obstante, la variación en las instituciones informales no es el único modo de distinguir la heterogeneidad de las trayectorias neopatrimoniales. Como se ha adelantado en la introducción, el grueso de las tipologías combinan la estructura de la autoridad estatal (desde la racionalidad legal y burocrática a la dominación patrimonial) con el nivel de competición política de un régimen (Fisun, 2007; Guliyev, 2011) ${ }^{1}$. Otros autores como Erdmann y Engel (2007) y Von Soest (2010) diferencian dentro del eje de dominación política (de racional/legal a patrimonial) entre las estructuras de Gobierno y $\triangleright$

\footnotetext{
${ }^{1}$ La tipología de Fisun trabaja sobre la teoría del sultanismo de Chehabi y Linz's (1998), para diferenciar entre neopatrimonialismo sultanista, oligárquico y burocrático. Sin embargo, la distinción de Guliyev dentro de las estructuras de autoridad estatal (régimen personalista, patronazgo institucional y el legal-burocrático) es terminológicamente más flexible. Siendo especialmente más apropiada cuando se analizan Estados que, como el kirguís, son difíciles de etiquetar como sultanistas o como oligárquicos, pero que encajan en la categoría de patronazgo institucional.
} 
las administrativas o burocráticas mientras mantienen el continuum autoritarismo-democracia liberal. Así, mientras en el primer eje básicamente se discrimina entre regímenes competitivos, semicompetitivos y poco competitivos (Fisun, 2007) ${ }^{2}$, el segundo eje supone un paso adelante en la elaboración de una tipología del neopatrimonialismo al agregar la concentración del poder en el Gobierno y el grado de separación de los gobernantes respecto a los medios legales de gobierno (Fisun, 2007).

Cualquier gobernante que aspire a la estabilidad trata de equilibrar los intereses de los actores más influyentes, sean aliados o no. Todo proceso de concentración de poder lleva implícito uno de reparto del poder. La labor de equilibrio de intereses ligada a la concentración de poder se puede desarrollar en, al menos, dos posibles dimensiones. Por un lado, en el grado de inclusividad o exclusividad presente en las instituciones centrales de gobierno en relación con el acomodo de los intereses de redes informales $u$ otros actores políticos (Iljamov, 2007; Ruiz Ramas, 2013). Von Soest (2010) o Fisun (2007) condensan la casuística de este rasgo en dos categorías, un régimen personalista o uno oligárquico. Por otro lado, se hace necesario especificar entre un clientelismo disperso (prebendalismo o «régimen [neo]patrimonial descentralizado» en Lewis, 1996) y uno más centralizado (Guliyev, 2012). Identificamos así dos variables -la concentración del poder en el gobernante y el nivel de centralización del clientelismo- muy útiles como punto de partida para estudiar la diversificación del poder en un Estado neopatrimonialista. Pero estas variables pertenecen a unidades de análisis más amplias tales como la creación de autoridad estatal y la fragmentación territorial del poder que necesitan ser complementadas por otros conceptos provenientes de la literatura de la construcción y la institucionalización del Estado. Además, cuando las fuentes de transformación de un Estado

\footnotetext{
${ }^{2}$ La mayoría de académicos consideran que el neopatrimonialismo puede persistir en regímenes autoritarios o híbridos, pero no en democracias liberales. Sin embargo, hay algunas excepciones como Pitcher et.al. (2009).
}

aumentan, superando la dimensión de la competitividad política, se entiende justificado cambiar el foco de la tipología desde el régimen político a las disparidades del Estado.

\section{La perspectiva de la construcción del Estado y Asia central}

La construcción del Estado es el proceso por el cual se establece «la autoridad par» (GrzymalaBusse y Jones Luong, 2002). Esta definición, que enfatiza la autoridad estatal y la capacidad de hacer cumplir las leyes, actualiza el influyente trabajo de Mann (1986) en el que se distingue el poder despótico del Estado, basado en el control de la toma de decisión, de su poder infraestructural, basado en su poder de implementación. El estado de la cuestión hasta Mann (1986) estaba dominado por quienes abogaban por «el regreso del Estado a primer plano", enfatizando el rol del Estado en determinar las decisiones políticas, esto es, de quienes conciben el Estado como un actor relativamente autónomo de clases sociales específicas (Krasner, 1978; Skocpol, 1979; Nordlinger, 1981; Tilly, 1975). Esta comprensión de la autonomía del poder estatal fue conceptuada y aislada por Mann como el «poder despótico del Estado», mientras en paralelo subrayó el poder infraestructural que cualquier Estado necesita para implementar sus políticas. En sus propias palabras «la capacidad del Estado para, de hecho, penetrar en la sociedad civil, y para implementar logísticamente decisiones políticas a través de su esfera de influencia» (Mann, 1986).

De esta distinción emerge un enfoque centrado en las capacidades del Estado ${ }^{3}$. En Migdal $\triangleright$

\footnotetext{
${ }^{3}$ En un primer momento por las capacidades del Estado se hacía referencia a las funciones del primer Estado moderno de Administración territorial, poder coercitivo y militar, la extracción de ingresos vía impuestos y posteriormente sus funciones intervencionistas en la economía y la sociedad. Con esas herramientas algunos investigadores comenzaron a medir la capacidad del Estado en términos de Estados fuertes y Estados débiles (Migdal, 1988; Jackman, 1993). El siguiente paso fue explicar distintos resultados dentro de un mismo Estado, abandonando así la exclusividad de la aplicación del concepto a nivel macro. Los comparativistas avanzaron en tres direcciones: elección racional (Geddes, 1994), el enfoque del «Estado en la sociedad» (Migdal et. al., 1994) y el neodesarrollismo (Jackman, 1993).
} 
et. al. (1994) se propone el enfoque del «Estado en la sociedad» a fin de desagregar el Estado como unidad de análisis. Se conceptúa al Estado como unidad multinivel con cuatro estratos: liderazgo central, Administración central, Administración regional y las oficinas sobre el terreno. Cada estrato contiene su propia arena dentro de la cual se disputan batallas por el poder entre actores estatales que compiten entre sí, al igual que entre actores estatales y no estatales ${ }^{4}$. De este modo, al referirse al poder estatal se habla de la autonomía para crear una «autoridad normativa vinculante» (Mann, 1986), pero también autonomía para ejecutar esas normas en el conjunto del territorio estatal sin caer en ninguna forma involuntaria de «migración de autoridad».

\subsection{Construcción del Estado en el Asia central postsoviética}

La primera generación de estudios sobre la construcción del Estado en el Asia central postsoviética (Cummings, 2000; Busse y Jones Luong, 2002; Ganev, 2005; Fritz, 2007) acentúa cinco atributos principales, si bien el desarrollo reciente de los acontecimientos en ese espacio desafía la plena validez de algunos de ellos. Primeramente, un elemento incontestable es la estrategia de los gobernantes para legitimar su autoridad por medio de vincular la construcción de la nación (nation-building) a la propia estatalidad. Para ello, según Cummings (2010) cinco técnicas de legitimación fueron utilizadas: una revisión de la historiografía, el simbolismo visual, el lenguaje, la indigenización del poder (aunque etnificación sería más preciso), así como otras formas de asociación entre la indigenización y el territorio. En segundo lugar, los presidentes centroasiáticos concentraron altas cotas de poder en los años noventa del

\footnotetext{
4 En una variante del enfoque «Estado en la sociedad», Peter Evans desarrolló un trabajo muy influyente donde él concibió el concepto de «autonomía enraizada» para explicar por qué algunos Estados en desarrollo son más exitosos que otros en alcanzar el crecimiento industrial (Evans, 1995 y 2013).
}

siglo pasado, bien vía reformas constitucionales, bien traspasando sus propias competencias definidas constitucionalmente. Como ha destacado Hale (2006), la especifidad del marco institucional postsoviético condujo a la consolidación de «presidencias patrimoniales». Las políticas de privatización y las relaciones centro-periferia conformaron la naturaleza de las políticas de patronazgo en estos Estados, siendo elementos clave para la sostenibilidad de una presidencia patrimonial mantener el equilibrio entre las élites tanto en el acceso a los recursos económicos a través del Estado como en la capacidad de ejercer autoridad en los niveles local y regional.

En tercer lugar, la debilidad del Estado en términos de capacidades infraestructurales se asoció al concepto de «Estado capturado», el cual se justifica debido a la apropiación privada de lo público. Un Estado capturado sirve a «los intereses de unos pocos captores, más que a la sociedad en su conjunto" (Fritz, 2007), pero al mismo tiempo carece de la «autonomía enraizada» (embedded autonomy) socialmente, que un Estado necesita, según Evans (1995), para su desarrollo y eficiencia institucional. Ganev (2005) explica que el proceso postsoviético ha sido el inverso al de la explicación clásica de la formación del Estado moderno de Charles Tilly. En ella, el Estado extrae recursos de la sociedad y a cambio, si bien involuntariamente, provee servicios e infraestructuras. Para Ganev lo ocurrido en los Estados postsoviéticos es una «extracción del Estado» por élites depredadoras favorecidas por un contexto institucional específico (la desintegración de una economía estatalizada) que promueve la conducta depredadora de los agentes estatales.

Dado que se extrae del Estado y no de otros grupos sociales, el proyecto de la élite depredadora se benefició de una resistencia social muy contenida. Como resultado de ello, las élites dirigentes carecieron de incentivos para invertir tanto en la administración de esa extracción a la sociedad como en las capacidades infraestructurales compensatorias. Sin embargo, acabada la fase inicial $D$ 
del cambio sistémico, la extracción ya no solo se concentra en el Estado, sino que también afecta a diversos grupos sociales. A la clase empresarial lo hace a través de impuestos pero también por medios ilícitos como el soborno o el raiderstvo ${ }^{5}$ (Junisbai, 2010). La clase trabajadora paga igualmente impuestos, mientras mantiene condiciones y salarios que no reflejan las plusvalías producidas por su labor, ofreciendo un desequilibrio significativo entre el coste de salarios y el beneficio de empresas privadas, pero también estatales o mixtas. Cierto es que, en paralelo, se ha experimentado un tímido amanecer de la resistencia social a la conducta depredadora de los agentes estatales. La inseguridad en el mundo de los negocios y el declive de las condiciones de vida alimentaron las revoluciones de 2005 y 2010 en Kirguistán, pero también los trágicos disturbios en Andiján (Uzbekistán), al tiempo que en Kazajstán la estrategia neodesarrollista de Nazarbayev no ha evitado desafíos al Estado tanto de empresarios como de trabajadores.

En cuarto lugar, la primera generación de estudios de construcción del Estado en Asia central (Busse y Jones Luong, 2002; Ganev, 2005) señala que la competición por la autoridad para formular políticas (policy-making) no fue solo un conflicto intraélites sino también intraestatal. Esta batalla por el poder enfrentó fundamentalmente a los actores estatales del centro y de las regiones sin participación de actores no estatales. Hasta cierto punto, esto fue consecuencia de una distinción ambigua entre el Estado y la sociedad heredada del periodo soviético (Busse y Jones Luong, 2002). Sin embargo, hoy en día la competición por la autoridad no es solo un juego entre agentes estatales. La emergencia de una élite económica autónoma como resultado de la liberalización de la economía por un lado, y de la creciente relevancia política de redes controlando tráficos ilegales

\footnotetext{
${ }^{5}$ El concepto de reidertsvo se refiere a la apropiación hostil e ilícita de una propiedad privada. Comúnmente se produce cuando personas cercanas a agentes gubernamentales son ayudados por los cuerpos de seguridad para forzar que empresarios vendan sus activos y bienes a un precio muy por debajo de su valor de mercado.
}

por otro lado, han añadido pluralidad a esa competición.

Finalmente, el declive del poder infraestructural del Estado tras la independencia afectó no solo a las competencias funcionales sino también a la capacidad de la autoridad central para impedir la «migración de autoridad» a las entidades regionales (Libman, 2012). Esto sucedió en procesos de baja conflictividad y dotados de reversibilidad, como la descentralización informal, pero también mediante enfrentamientos por ejercer la autoridad sobre un territorio de alta intensidad, como el desafío al monopolio del Estado en el uso de la violencia en Tayikistán durante y después de la guerra civil. Por todo ello, se considera que el enfoque de Migdal (1994) encaja perfectamente en el contexto centroasiático en tanto que el Estado no es un actor unitario y por tanto necesita ser desagregado (Busse y Jones Luong, 2002).

\section{Analizando la diversificación del poder}

Como se ve en la Tabla 1 se propone un marco para analizar la diversificación del poder en un Estado mediante el examen cualitativo de un número de indicadores que representan la estructura de toma de decisiones en las instituciones centrales de gobierno y el grado de fragmentación territorial del poder.

Atendiendo a las características anteriores, en el análisis de la heterogeneidad en los Estados neopatrimonialistas de Asia central se distinguen tres niveles de diversificación del poder genéricos:

1. Diversificación del poder mínima. Poder hegemónico, control exclusivo de las instituciones y ausencia de desafíos significativos a la implementación de las decisiones del centro en las regiones (Turkmenistán).

2. Diversificación del poder limitada. El poder del Estado es altamente concentrado. El presidente es autoritario pero todavía requiere de acuerdos de reparto del poder (power-sharing agreements) con otras redes de élites o carece de control total sobre el conjunto del territorio. (Uzbekistán/Kazajstán y Tayikistán). 


\section{LA DIVERSIFICACIÓN DEL PODER EN LOS ESTADOS NEOPATRIMONIALISTAS DE ASIA CENTRAL}

TABLA 1

INDICADORES DE ANÁLISIS DE LA DIVERSIFICACIÓN DEL PODER

\begin{tabular}{|c|c|}
\hline $\begin{array}{l}\text { 1. Grado de concentración de la toma de decisiones en las } \\
\text { instituciones del Gobierno central }\end{array}$ & 2. Fragmentación territorial del poder (Estado en las regiones) \\
\hline $\begin{array}{l}\text { 1.1. Autonomía presidencial } \\
\text { 1.1.1. Inclusión o exclusión de élites y redes informales } \\
\text { alternativas en las instituciones de gobierno centrales. } \\
\text { Existencia de coaliciones de reparto de poder. Distribución } \\
\text { de 1) servicios públicos y acceso a recursos, y 2) cargos } \\
\text { ministeriales y otros puestos en la Administración } \\
\text { 1.1.2. Inclusividad o cooptación en las instituciones formales } \\
\text { (Parlamentos y partidos del poder, oficialistas) } \\
\text { 1.1.3. Fuente de autoridad en las redes informales de élites y } \\
\text { grado de control recíproco entre patrón y cliente } \\
\text { 1.2. Relaciones Estado-sociedad (competición entre élites estatales, o } \\
\text { entre actores estatales contra no estatales) } \\
\text { 1.3. Relación élite-masas }\end{array}$ & $\begin{array}{l}\text { 2.1. Estructura legal de las relaciones territoriales entre el centro y las } \\
\text { regiones } \\
\text { 2.2. Formas de descentralización informal: } \\
\text { 2.2.1. Necesidades del Estado del apoyo de las redes informales } \\
\text { locales para llevar a cabo las normativas emanadas del } \\
\text { Gobierno central } \\
\text { 2.2.2. Ausencia de control administrativo vertical sobre el conjunto } \\
\text { del territorio (manipulación de la implementación de las } \\
\text { decisiones gubernamentales por agentes locales) } \\
\text { 2.2.3. Descentralización informal (fiscal, elaboración de legislación } \\
\text { alternativa, etcétera) } \\
\text { 2.3. Ausencia del monopolio de la violencia en partes del territorio } \\
\text { estatal }\end{array}$ \\
\hline
\end{tabular}

TABLA 2

DIVERSIFICACIÓN DEL PODER EN ASIA CENTRAL

\begin{tabular}{|l|c|c|c|}
\hline \multicolumn{1}{|c|}{ Concentración de la toma de decisiones } & Alta & Mragmentación territorial del poder & Baja \\
\hline Hegemónica & & & TKM \\
\hline Alta & TJK & UZB/KAZ & \\
\hline Baja & KGZ & & \\
\hline Fuente: elaboración propia. & & & \\
\hline
\end{tabular}

3. Poder moderadamente diversificado. Formalmente el poder está todavía altamente concentrado pero se necesitan incorporar acuerdos estables de reparto del poder con múltiples redes de élites u otros actores políticos, no solo con un número limitado de ellos. Igualmente, esta categoría incluye a países donde el control sobre el territorio del Estado es desafiado (Kirguistán).

\section{Aplicación del marco teórico sobre los casos de Asia central}

\subsection{Turkmenistán: hegemonía en la toma de decisiones y alta centralización}

Turkmenistán representa uno de los polos en Asia central respecto a la diversificación del poder. De lejos es el Estado donde la toma de decisiones está más concentrada y la centralización menos desafiada. Tras la desintegración de la URSS, el entonces primer secretario del Partido Comunista, Saparmurat Niyazov, maniobró eficazmente para instaurar un régimen centralizado dotado de un extremo culto a la personalidad.

La concentración del poder en manos de Niyazov fue absoluta. Además de presidente, él era primer ministro, encabezaba el único partido político permitido y controlaba la selección de miembros del Halk Maslahaty (Consejo Popular). Niyazov con el apoyo del Halk Maslahaty modificó la constitución en diciembre de 1999 para establecer una presidencia vitalicia. Los otros poderes del Estado, el legislativo (Majlis) y el poder judicial, fueron subordinados a la autoridad presidencial (Pomfret, 2012). El culto a la personalidad alcanzó cotas difíciles de igualar. Niyazov asumió el nombre de Turkmenbashi el Grande, líder de los turkmenos, hizo levantar monumentos megalómanos y redactar normas bizarras tales como renombrar los meses del año con los de miembros de su familia o hacer lectura obligatoria en escuelas, universidades y agencias gubernamentales el Ruhnama. Una obra escrita por Niyazov que combina la autobiografía, la guía espiritual y el revisionismo histórico con un cariz nacionalista. 
Con la muerte de Niyazov en 2006, asumió la presidencia Gurbangulí Berdimujamédov, quien impulsó dos cambios relevantes respecto a la distribución del poder. Por un lado, incrementó el rol de su círculo familiar en las instituciones centrales de gobierno y agencias estatales con acceso a recursos energéticos. Por otro lado, implementó una nueva política regional orientada a dotar de mayor autonomía a las élites locales que, según Kunysz (2012), puede conducir a la consolidación de redes de patronazgo regionales.

\subsection{Kirguistán: pluralismo en un Estado fragmentado}

Kirguistán no es solo el polo opuesto de Turkmenistán en términos de diversificación del poder en Asia central, sino que también es el único caso donde la liberalización política ha avanzado -con algunas interrupciones- a niveles de autoritarismo competitivo (Levitsky y Way, 2010). Asumiendo que Kirguistán nunca ha sido «la isla de la democracia» como se le acuñó en los años noventa, es cierto que la liberalización de la arena política, de los medios y el desarrollo de la sociedad civil, definitivamente hacen del país una ínsula en el océano de autoritarismo que es Asia central.

A pesar de ello, la competitividad política en Kirguistán ha experimentado cambios constantes en los últimos veinte años. Principalmente es debido a los intentos de dos presidentes, Askar Akayev y Kurmanbek Bakiyev, por acumular poder en detrimento de las expectativas de otras élites y opciones políticas. En Kirguistán, al margen de operar en un contexto institucional informal, estas redes de élites poseen cuatro rasgos principales. Primero, se vertebran de manera local y se benefician del apoyo social de la comunidad en un intercambio por prestaciones materiales, pero también gracias a inversiones morales. Segundo, las redes informales compiten y establecen alianzas entre sí, bien para desafiar al Estado, o bien para promocionarse dentro de él. Tercero, incorporan actores no estatales, especialmente «élites autónomas» (Radnitz, 2011), élites económicas surgidas con la privatización y la liberalización, pero también a menudo figuras enriquecidas gracias a actividades criminales (Radnitz, 2011). Finalmente, tienen capacidad para acceder a cargos electos tanto como candidatos independientes como penetrando en partidos políticos establecidos o de nuevo cuño (Ruiz Ramas, 2013).

Estas redes informales de ámbito local controlan recursos económicos dentro de sus distritos, pero compiten entre sí por incrementar su influencia política y acceso a recursos de mayor volumen. Como resalta Engvall (2011), la élite económica se introduce en la política para extraer recursos del Estado y alcanzar la protección legal asociada a algunos cargos públicos. De esta manera, el Estado en Kirguistán es un mercado donde los puestos son adquiridos a través del soborno y las conexiones. El resultado es un Estado donde la competición por la inclusión en la toma de decisiones en el nivel central permanece viva y la concentración de poder es frágil, pero sobre todo donde la fragmentación territorial de la autoridad es profunda, multicausal y permanentemente en renegociación (Ruiz Ramas, 2013).

\subsection{Tayikistán: un ejecutivo central irrestricto en un Estado altamente fragmentado}

Tayikistán es el único caso de Asia central que posee una alta concentración ejecutiva en las instituciones centrales del Estado y al mismo tiempo elevados niveles de fragmentación territorial. Si bien es cierto que el presidente Emomali Rahmon y su red de Dangara en el distrito de Kulob ocupan una posición hegemónica, marginando a élites y actores en las instituciones centrales, también lo es que su poder es frágil más allá de Dushanbé y Kulob (Engvall, 2006; McGlinchey, 2012).

Comúnmente el régimen político tayiko es categorizado como plenamente autoritario (Mateeva $\triangleright$ 
2009; Finkel, 2012). Como presidente del país, Rahmon disfruta de una amplia autonomía en el proceso de toma de decisiones hasta el punto de ser categorizado su régimen por distintos investigadores como uno plenamente autoritario. Esto es resultado de dos movimientos de Rahmon. Primero, impidió la implementación de los mecanismos de reparto del poder concertados con las fuerzas de la coalición Oposición Tayika Unida (UTO) durante los Acuerdos de Paz de 1997. Segundo, apartó progresivamente de las instituciones centrales a élites aliadas durante la guerra, especialmente aquellas procedentes de Khodjent (entonces Leninabad) en el norte de la provincia de Sughd. Para garantizar su autoridad en las regiones, a pesar de haber privado a las organizaciones de la UTO de sus cuotas de poder, Rahmon alcanzó acuerdos informales bilaterales con excomandantes de la UTO, algunos directamente considerados señores de la guerra.

Ese tipo de acuerdos fueron especialmente relevantes en el Valle del Rasht y en la Provincia Autónoma de Alto Badajshán. Funciones estatales básicas, como el control de fronteras, han dependido de la colaboración con estos comandantes, algunos de los cuales fueron integrados en las fuerzas de seguridad del Estado. Posteriormente, en casos particulares, Rahmon trató de renegociar dichos pactos e incluso reemplazar a varios excomandantes. Movimientos replicados con una férrea resistencia de los caudillos y sus comunidades locales hasta el punto de conducir a enfrentamientos armados e incluso a la ocupación del territorio por el ejército bajo la ley marcial.

Se concluye, por tanto, que la competición por ejercer la autoridad estatal permanece en determinadas regiones en la forma de lucha entre élites donde toman parte actores estatales y no estatales. Caudillos locales, antiguos señores de la guerra y barones de la droga compiten entre sí, dentro y fuera del Estado, al tiempo que intentan resistir las presiones de Dushanbé. Aunque las masas no han tenido hasta el momento un rol independiente, el apoyo de las comunidades locales ha resultado decisivo en las batallas por la autoridad estatal, en particular, en los desafíos a la autoridad central. Al margen de la contienda intraélite por el poder y el acceso a recursos, las relaciones Estado-sociedad están afectadas, y pueden estarlo más en el futuro cercano, por la capacidad de movilización de la religión y las redes interpersonales y comunales que la religión ayuda a establecer.

\subsection{Kazajstán: ¿hacia la consolidación de una oligarquía neopatrimonialista?}

Nursultan Nazarbayev, quien ya gobernaba Kazajstán en el periodo soviético, ha reunido durante su largo mandato una gran cuota de poder ejecutivo y potestad sobre los principales recursos económicos, destacando los hidrocarburos. En especial desde 1994, Nazarbayev maniobró exitosamente para fortalecer la presidencia respecto al parlamento avanzando hacia un sistema de gobierno hiperpresidencialista. En paralelo, su círculo familiar más cercano aceleró la acumulación de activos económicos, asegurándose de igual modo una posición privilegiada ante nuevas oportunidades de negocios en los que mediase capital extranjero.

Al margen de estos dos procesos fácilmente trazables empíricamente, las instituciones kazajas han promovido sin descanso un culto a la personalidad de Nazarbayev con puntos culminantes tales como ser nombrado por el parlamento «Líder de la Nación». Honor que lleva implícita la inmunidad vitalicia ante cualquier tipo de acusación. Sin embargo, representar a Kazajstán como un Estado donde un hombre posee un control vertical e ilimitado sobre las arenas política y económica no encaja con la realidad. Ninguna decisión esencial puede ser tomada en Kazajstán sin la aprobación de Nazarbayev, pero en cada periodo desde la independencia ha habido un número de redes de élites capaces de influenciar las decisiones de las instituciones centrales e incluso $D$ 
de desafiar abiertamente la autoridad central cuando han entendido que sus intereses no estaban siendo atendidos. En paralelo, la alta concentración de poder en las instituciones centrales no ha impedido ni una indeseada descentralización territorial ni que élites regionales ampliasen su autonomía aprovechando su control sobre las inversiones extranjeras en el sector energético.

A medida que la presidencia de Nazarbayev, de 74 años, va llegando a su fin, y no se cuenta ni con un claro sucesor ni con una figura capaz de sustituir el liderazgo de Nazarbayev, no se puede excluir el estallido de encarnizadas luchas por el poder entre las principales redes informales de élites. Siendo esto cierto, existen factores estructurales e institucionales que permiten prever, por el contrario, la neutralización de ese choque a través de un pacto orientado a la instauración de un neopatrimonialismo oligárquico más o menos inclusivo. Entre los factores estructurales destaca la necesidad de estabilidad para mantener la calma en los inversores foráneos. A nivel institucional, en los últimos años se identifica una tendencia hacia la consolidación de un neopatrimonialismo oligárquico ${ }^{6}$. Por un lado, en cuanto al marco político, en el nivel central destaca la implementación de compromisos e iniciativas para acomodar una pluralidad de élites en instituciones donde compartir poder y acceso a recursos. Entre ellas destacan el Parlamento o el partido oficialista Nur Otan (Ostrowski, 2009). En la esfera económica se cumple ese mismo objetivo, el de la inclusión de las principales agrupaciones políticas informales, con la creación de holdings estatales tales como Samruk-Kazyna. Por último, la oligarquización kazaja se afianza con el ya mencionado robustecimiento de las élites regionales.

Con todo, no deben descartarse presiones significativas contra la oligarquización del Estado kazajo favorables a una mayor democratización

\footnotetext{
${ }^{6}$ Según Fisun (2003:6) el neopatrimonialismo oligárquico está «vinculado con la formación de un amplio estrato de actores rentistas neopatrimonialistas, actuando juntos con/o en lugar de las instituciones gubernamentales, vía redes de patronazgo e intercambios clientelares".
}

desde sectores distintos a las élites. Pequeños y medianos empresarios así como trabajadores han dado muestras ya por distintos medios de estar preparados para luchar por sus propios intereses colectivos. La importancia creciente del movimiento obrero en Kazajstán en los últimos años permaneció desconocida para la mayoría de analistas hasta la trágica represión de la huelga de los trabajadores del sector petrolero en Zhanaozen en diciembre de 2011, cuando al menos 15 manifestantes desarmados fueron asesinados por las autoridades ${ }^{7}$.

\subsection{Uzbekistán: alta concentración del poder de decisión y débil capacidad de implementación en las regiones}

El Estado uzbeko se caracteriza principalmente por tener una estructura en el poder ejecutivo altamente centralizada; unas fuerzas de seguridad sólidas que ya han combatido desafíos al monopolio del Estado en el uso de la violencia e intentos de acometer severos procesos de fragmentación territorial del poder (Namagán, 1991-1992; acciones del Movimiento Islámico de Uzbekistán, Andiján, 2005); y finalmente por un débil poder infraestructural que ha favorecido una progresiva devolución informal del poder a actores locales en determinadas regiones. Islam Karimov, como cúspide de un sistema neopatrimonial, disfruta de una gran discrecionalidad en el ejercicio de su Gobierno. A pesar de ello, su poder ejecutivo es constreñido por la incapacidad para implementar la autoridad central en todo el territorio sin alcanzar acuerdos informales con élites locales. Estas élites ganan a cambio autonomía ejecutiva en sus regiones de decisión y recursos para crear redes de patronazgo perdurables en el tiempo. De tal modo, el Estado neopatrimonial consolidado con Karimov combina una fuerte consolidación de poder político $\triangle$

\footnotetext{
${ }^{7}$ Más información sobre los disturbios en Zhanaozen en: Rustemova, Assel $(2011,2012)$ Kazakhstan, November/December 2011, January, March 2012 and May/June 2012 issues of the Central Asian Observatory Bulletin. Artículos disponibles en http://www.asiacentral.es/boletin.php
} 
y económico en el círculo cercano al presidente, con múltiples escenarios de competición por la autoridad, donde élites regionales han visto fortalecida su posición. Dos ejemplos de ello son las competiciones por la composición del Parlamento (Iljamov, 2004), y por las designaciones de los ejecutivos regionales (Markowitz, 2012).

Así mismo, existen también disputas entre distintas redes de élites en las instituciones centrales donde Karimov ha tomado el papel de árbitro. Durante años la lucha más relevante fue entre quienes encabezaban las dos principales instituciones de seguridad interna: el Ministerio del Interior y los Servicios de Seguridad Nacional (SSN). Intentando amortiguar el impacto de las luchas intraélites, antes y después de su sucesión, Karimov parece dispuesto a fortalecer al poder legislativo respecto al ejecutivo, anticipando con ello un contexto institucional dotado de mayor reparto de poder. $Y$ es que como en el caso de Nazarbayev, la avanzada edad de Karimov (77) predispone a preparar su sucesión y a prever nuevos conflictos entre las élites con influencia en Taskent. De momento, existe una batalla por el poder, en la que destacan las alianzas encabezadas por el primer ministro Shavkat Mirziyayev y el ministro de finanzas Rustam Azimov. Este último es hoy el máximo favorito a suceder a Karimov, incluso a pesar de haber protagonizado un enconado enfrentamiento con la hija de éste, Gulnora Karimova, tras el cual ésta ha sido defenestrada hasta por su propio padre.

\section{Bibliografía}

[1] BALMACEDA, M. (2008). Energy Dependency, Politics and Corruption in the Former Soviet Union. Russia's Power, Oligarchs' Profits and Ukraine's Missing Energy Policy 1995-2006. Londres: Routledge.

[2] BRATTON, M. y VAN DE WALLE, N. (1997). Democratic Experiments in Africa. Regime Transitions in Comparative Perspective. Cambridge: Cambridge University Press.
[3] CAROTHERS, T. (2002). «The End of the Transition Paradigm». Journal of Democracy, vol. 13, $\mathrm{n}^{\circ} 1$ (January), pp. 5-21.

[4] COLLINS, K. (2006). Clan Politics and Regime Transition in Central Asia. Cambridge: Cambridge University Press.

[5] COLLINS, K. (2009). «Economic and Security Regionalism Among Patrimonial Authoritarian Regimes: The Case of Central Asia». EuropeAsia Studies, vol. 61, n², pp. 249-281.

[6] CUMMINGS, S. (2005). Kazakhstan: Power and Elite. London and New York: I. B. Tauris.

[7] EISENSTADT, S. N. (1973). Traditional Patrimonialism and Modern Neopatrominialism. Londres, Beverly Hills/CA: Sage Publications.

[8] ERDMANN, G. y ENGEL, U. (2006). «Neopatrimonialism Revisited - Beyond a CatchAll Concept». GIGA Working Paper, $\mathrm{n}^{\circ} 16$. Hamburg: German Institute of Global and Area Studies (GIGA).

[9] ERDMAN, G. (2012). «Neopatrimonialism: Problems of a Catch-All Concept». En Stewart, S. (ed). Presidents, Oligarchs and Bureaucrats: Forms of Rule in the Post-Soviet Space. London: Ashgate Publishing, Ltd., p. 258.

[10] FINKEL, E. (2012). «Democratization No more Colour! Authoritarian Regimes and Colour Revolutions in Eurasia». Democratization, vol. 19, n¹, pp. 1-14.

[11] FISUN A. (2007). «Postsovetskie Neopatrimonial' nye Rezhimy: Genezis, Osobennosti, Tipologija». Otechestvennye zapiski, $\mathrm{n}^{\circ} 6$.

[12] GANEV, V. I. (2005). «Post-Communism as an Episode of State Building: A Reversed Tillyan Perspective». Communist and Post Communist Studies, vol. 38, n 4, pp. 425-445.

[13] GULIYEV, F. (2011). «Personal rule, Neopatrimonialism, and Regime Typologies: Integrating Dahlian and Weberian Approaches to Regime Studies». Democratization, vol. 18, n 3 , pp. 575601.

[14] HALE, H. E. (2006).«Democracy or Autocracy on the March? The Colored Revolutions as Normal Dynamics of Patronal Presidentialism». Communist and Post-Communist Studies, $\mathrm{n}^{\circ} 39$, pp. 305329.

[15] HALE, H. E. (2011). «Formal Constitutions in Informal Politics. Institutions and Democratization in Post-Soviet Eurasia». World Politics, vol. 63, $\mathrm{n}^{\circ} 4$, pp. 581-617. 
[16] ILJAMOV, A. (2007). «Neopatrimonialism, Interest Groups and Patronage Networks: the Impasses of the Governance System in Uzbekistan». Central Asian Survey, $n^{\circ} 26$, p. 65.

[17] JONES LUONG, P. (2002). Institutional Change and Political Continuity in Post-Soviet Central Asia: Power, Perceptions, and Pacts. Cambridge: Cambridge University Press.

[18] JUNISBAI, B. (2010). «A Tale of Two Kazakhstans: Sources of Political Cleavage and Conflict in the Post-Soviet Period». Europe-Asia Studies, vol. 62, $\mathrm{n}^{\circ} 2$, pp. $235-269$.

[19] KONONENKO, V. y MOSHES, A. (2011). Russia as a Network State: What Works in Russia When State Institutions Do Not?. London: Palgrave Macmillan.

[20] LEVITSKY, S. y WAY, L. (2010). Competitive Auhtoritarianism. Hybrid Regimes After the Cold War. Cambridge: Cambridge University Press.

[21] LEWIS, P. (1996). «From Predendalism to Predation: The Political Economy of Decline in Nigeria». The Journal of Modern African Studies, vol. 34, $n^{\circ} 1$, pp. 79-103.

[22] LEWIS, D. (2008). The Temptations of Tyranny in Central Asia. New York: Columbia University Press.

[23] LIBMAN, A. (2009). "Government-Business Relations in Central Asia: Approaches to Comparative Analysis». Russian Studies, $n^{\circ} 5$, pp. 57-70.

[24] LYNCH, D. (2013). «Greed, Lust, War and Peace: The 2012 Battle for Khorog, Tajikistan». Paper presented at the ASN World Convention. Columbia University, pp. 18-20, April 2013.

[25] MÉDARD, J. F. (1982). «The Underdeveloped State in Tropical Africa: Political Clien- telism or Neo-Patrimonialism?», In: Clapham, C. (ed.). Private Patronage and Public Power. Political Clientelism in the Modern State (pp. 162-192). New York, NY: St. Martin's Press.

[26] MØLLER, J. y SKAANING, S. (2010). «PostCommunist Regime Types: Hierarchies Across Attributes and Space». Communist and PostCommunist Studies, vol. 43, pp. 51-71.

[27] MURPHY, J. (2006). «lllusory Transition Elite Reconstruction in Kazakhstan». Europe-Asia Studies, vol. 58, nº 4, pp. 523-554.
[28] OSTROWSKI, W. (2009). «The Legacy of the 'Coloured Revolutions': The Case of Kazakhstan». Journal of Communist Studies and Transition Politics, vol. 2, n 3, pp. 345-368.

[29] OSTROWSKI, W. (2010). Politics and Oil in Kazakhstan. London: Routledge.

[30] PITCHER, A.; MORAN, M. y JOHNSTON, M. (2009). «Rethinking Patrimonialism and Neopatrimonialism in Africa». African Studies Review, vol. 52, no 1, pp. 125-156.

[31] RADNITZ, S. (2006). "What Really Happened in Kyrgyzstan?». Journal of Democracy, vol. 17, $\mathrm{n}^{\circ}$ 2, pp. 132-146.

[32] RUIZ RAMAS, R. (2012). "The Institutional Persistence of Patrimonialism in the Kyrgyz Republic: Testing a Path Dependency (19912010)». En Ahrens, J. and Hoen, H. (ed.). Institutional Reform in Central Asia: PoliticoEconomic Challenges. Londres: Routledge.

[33] SATPAEV, D. (2007). «An Analysis of the Internal Structure of Kazakhstan's Political Elite and an Assessment of Political Risk Levels». En Uyama, T. (ed.). Empire, Islam, and Politics in Central Eurasia. Sapporo: Slavic Research Center, Hokkaido University.

[34] SCHATZ, E. (2004). Modern Clan Politics: The Power of 'Blood' in Kazakhstan and Beyond. Seattle: University of Washington Press.

[35] SNYDER, R. (1992). «Explaining Transitions from Neopatrimonial Dictatorships». Comparative Politics, vol. 24, $n^{\circ} 4$, pp. 379-400.

[36] STEWART, S. (2012). Presidents, Oligarchs and Bureaucrats: Forms of Rule in the Post-Soviet Space. London: Ashgate Publishing.

[37] TILLY, C. (1975). «Western-State Making and Theories of Political Transformation". The Formation of National States in Western Europe. Princeton: Princeton University Press.

[38] TUNÇER-KILAVUZ, I. (2011). "Understanding Civil War: A Comparison of Tajikistan and Uzbekistan». Europe-Asia Studies, vol. 63, n 2, pp. 263-290.

[39] WILSON, A. (2005). Virtual Politics. Faking Democracy in the Post-Soviet World. London: Yale University Press. 